\title{
Article
}

\section{Relation between Epidural Analgesia and severe perineal laceration in childbearing women in Catalonia}

Garcia-Lausin, L, Perez-Botella, Mercedes, Duran, X, RodríguezPradera, S, Gutierrez-Martí, M J and Escuriet, R

Available at http://clok.uclan.ac.uk/25750/

Garcia-Lausin, L, Perez-Botella, Mercedes ORCID: 0000-0003-3320-6446, Duran, X, Rodríguez-Pradera, S, Gutierrez-Martí, MJ and Escuriet, $R$ (2019) Relation between Epidural Analgesia and severe perineal laceration in childbearing women in Catalonia. Midwifery, 70 . pp. 76-83. ISSN 0266-6138

It is advisable to refer to the publisher's version if you intend to cite from the work. http://dx.doi.org/10.1016/j.midw.2018.12.007

For more information about UCLan's research in this area go to http://www.uclan.ac.uk/researchgroups/ and search for <name of research Group>.

For information about Research generally at UCLan please go to http://www.uclan.ac.uk/research/

All outputs in CLoK are protected by Intellectual Property Rights law, including Copyright law. Copyright, IPR and Moral Rights for the works on this site are retained by the individual authors and/or other copyright owners. Terms and conditions for use of this material are defined in the policies page.

\section{CLoK}

Central Lancashire online Knowledge www.clok.uclan.ac.uk

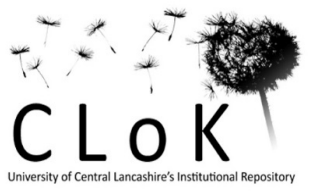


TITLE

"Relation between Epidural Analgesia and severe perineal laceration in childbearing women in Catalonia" 


\section{ABSTRACT}

Objective: Our objectives were to study the association between epidural analgesia and risk of severe perineal laceration (SPL), and identify additional risk factors for SPL. This multicentre study consisted of an analysis of data from the MidconBirth Phase I Dadabase, on the use of EA and perineal results during childbirth. (World Health Organization, International Clinical Trials Registry Platform,2016:http://apps. who.int/trialsearch/Trial2.aspx?TrialID=ISRCTN17833269).

Methods: We conducted a prospective study of pregnant women at term between July 2016 till July 2017 in 30 public maternity hospitals in Catalonia, Spain. Inclusion criteria were an uncomplicated singleton pregnancy, in cephalic presentation and vaginal birth. Data was analysed separately for instrumental births and spontaneous vaginal births, as the former is more frequently associated with episiotomy and more perineal lacerations. Risk factors as well as protective factors in each cohort of women (instrumental and spontaneous vaginal birth), were identified. Multivariate logistic regression model was performed to study the association between epidural analgesia and SPL to identify potential confounders. Odds ratios $(\mathrm{OR})$, using $95 \%$ confidence intervals $(\mathrm{Cl})$ were constructed.

Findings: During the study period, 5,497 eligible women gave birth, $77.46 \%$ of them received epidural analgesia. SPL occurred in $1.63 \%$ of births. The univariate analysis showed births with epidural analgesia had significantly higher rates of inductions, augmentation of labour, lithotomy position for birth and episiotomy. However, this association disappeared when the variable "type of vaginal birth" was introduced. In multivariate logistic regression, nulliparity was the major predictor for SPL (OR: 0.17; Cl 95\%: 0.08-0.34, p: 0.000).

Key conclusions: Epidural analgesia was not associated with SPL once confounding factors were included. Other interesting factors associated with SPL were identified.

Implications for practice: This paper identifies important practice areas which contribute to SPL and which have the potential to be rectified. It offers evidence on the role that EA plays on pelvic floor injuries and it adds to existing evidence about the disadvantages of using the lithotomy position for birth, especially in relation to SPL. It highlights the need for practice change in Catalonia from what can be considered a medical model of care to one more aligned with the midwifery philosophy of care through the development of clinical guidelines. 
It also signals the need to provide women with evidence base upon which to make informed choices on the use of EA, specifically in relation to SPL.

\section{INTRODUCTION}

Globally between 30-75\% of women sustain some degree of perineal trauma during birth. There is a wide variation in the reported incidence of severe perineal laceration (SPL) from childbirth being between $0.8 \%$ and $3 \%$ (Meister et al, 2016), although results from a systematic review shows an incidence as high as $11 \%$ (Duddling et al 2008). Severe perineal laceration is defined by the Royal College of Obstetricians and Gynaecologists as a tear extending into the deep transverse perineal muscles and fibres of the anal sphincter (third degree) or rectal mucosa (fourth degree) (RCOG, 2015). Injury to the genital tract during vaginal birth, can lead to adverse health outcomes for women such as severe pain, bleeding, infection, urinary and faecal incontinence, dyspareunia, pelvic organ prolapses and ultimately to severe psychological and social problems (Rathfisch, 2010). Short-and longterm health problems resulting from SPL can significantly affect women's quality of life (Gurol-Urganci, 2013).

SPL have been associated with various predisposing factors (Smith et al, 2013); nutritional status, maternal body mass index, primiparity (Rognant et al., 2012; Landy et al, 2011), precipitate labour, foetal weight $>4 \mathrm{~kg}$, instrumental delivery (with forceps presenting a higher risk than ventouse) ( Voldner et al., 2009; Prager et al., 2008), longer duration of second stage of labor (Groutz et al, 2010; Rognant et al., 2012), large for gestational age or birthweight (Groutz et al, 2010; Landy et al, 2011) and occipito posterior (OP) position, shoulder dystocia (Rognant et al., 2012; Groutz et al, 2010), gestational age > 40 weeks, Asian or Indian ethnicity, and kneeling or lithotomy position for birthing (Kalis et al, 2007).

The use of epidural analgesia during labor has also been identified by some studies as an extrinsic factors for SPL (Groutz et al., 2011). The reasons that may explain this include a serie of physiological processes and associated health professionals responses and interventions that can be considered part of the so called cascade of interventions (Lothian et al, 2015). EA interferes with the main hormones involved in the birth process, such as decreased oxytocin secretion, prostaglandin E2 or $\beta$-endorphins (Hawkins, JL, 2010). This hormone decrease leads to a longer labour, immobility, a greater need for stimulation with oxytocin for obstructed labor, lengthened second stage of labor (Sng et al, 2014) and 
increased incidence of foetal malposition during birth (occiput posterior and transverse positions) and the increased risk of instrumental birth (Jones et al, 2012; Anim-Somuah, 2018). On the other hand, (Simic et al., 2012; Landy et al, 2011) different studies reported a decreased association between SPL and EA use and identified EA as a protective factor to suffering an SPL. Reporting lower risk of SPL associated with EA use being explained by having more controlled crowning and delivery of the head with resulting fewer lacerations.

Furthermore, evidence on early initiation of EA and risk of instrumental birth seems to be contradictory also: some studies support using EA early in labor (before the foetus is well down in the pelvis) (Hasegawa et al., 2013; Pergialiotis et al, 2014 is associated with extension and/or difficulty in flexion of the foetal head, which interferes with rotation and descent and it increases instrumental births. However, a Cochrane Systematic Review (CSR) (Sng et al., 2014), concluded that EA does not need to be delayed because early initiation does not increase incidence of instrumental births and caesarean sections in primiparous women. Some limitations were found in the trials; the technique to assess dilatation was not exact and the participants of the study were only primiparous women in spontaneous labour thereby limiting the generalizability of the findings. In addition, the authors draw no firm conclusions from the CSR. In a recent CSR by Anim-Somuah et al. (2018), although an increase in intrumental birth when women had EA, a post hoc subgroup analysis showed this effect is not seen in recent studies and suggest that modern approaches to EA such Walking-Epidural in labor may help to reduce the association of epidural analgesia use with the incidence of instrumental vaginal births. Therefore, the association between EA and instrumental vaginal birth remains unclear and more studies are required.

This leads to the concern as whether EA during labor negatively impacts on perineal results at birth. EA has become an effective and popular method of pain control during vaginal birth. Even though the risks and side effects associated with EA are well known, demand for this method of pain relief in labor in developed countries is high. In recent years its use has extended significantly in such countries: $30 \%$ in the United Kingdom, 60\% in the USA, 30$69 \%$ in Canada, and in Spain it is used by $85-90 \%$ of women in labor (Anim-Somuah et al, 2011; Grant et al, 2015), being used by around $77 \%$ of women in labor in Catalonia. This high rate of EA use in Catalonia has prompted to question whether this rate influences an increase in SPL during labor in Catalonia. This concern is supported by the fact that the 
leading indication for instrumental birth is foetal malposition and ineffective pushing, and that EA prolongs the second stage of labor (Anim-Somuah et al, 2018).

The context of midwifery and obstetric practice in Catalonia, and in Spain in general follows a medical model with a rate of caesarean section of $25 \%$ [well above that the recommended $10-15 \%$ by international organizations (WHO, 2018)], and an incidence of induction of labor of around $20 \%$ (nearly doubles the recommended 10\%). This medicalized model of maternity care in Catalonia may be one of the reasons for the high incidence of EA use in childbirth. Recently, efforts have been made to improve this situation in Catalonia, The National Health System in Spain, created the Strategy for Assistance at Normal Childbirth (SANC) (Ministry of Health and Consumer's Affairs, 2007), the main goal of SANC was to establish specific recommendations on intrapartum care with the aim of improving its quality. In 2010 "clinical practice guide on normal birth care" was published to that effect. In 2012, an evaluation of the strategy showed no significant improvement in the quality of care provided and this generated a series of questions about intrapartum care provision which the MidconBirth (MCB) study aims to answer. MCB's goal is to analyse the relationship between midwife care provision during normal birth and a series of outcomes, including type of birth, type of onset of labor, use of different methods of pain relief during labour and perineal results after childbirth (Escuriet et al, 2017). We decided to conduct a new line of research to analyse the high rate of EA use in Catalonia, Spain ad its relationship to SPL. We aim to estimate whether EA carries an increased risk of SPL in women participating in MCB Study. Moreover, important maternal and birth characteristics were taken into account. Establishing whether EA in the presence of other obstetric factors predisposes to SPL has important consequences for decision making about childbirth pain relief use and potential long-term effects on maternal health and lifestyle.

\section{METHODS}

\section{Study design}


We conducted a multicentre prospective study of women giving birth a live infant in 30 maternity hospitals in Catalonia from July 2016 and July 2017. Data for this study were obtained from MBC study database (Escuriet, 2017).

\section{Study population}

Our study population included 5,497 from the 6,182 childbearing women participating in MCB study phase I. 669 women were excluded from the study as they had a caesarean section, and 16 were excluded as some of their data was incomplete. We compared maternal demographic and clinical factors. Inclusion and exclusion criteria are presented in Box 1.

Birth characteristics such as onset of labor, use of EA (as the major outcome variable), nationality, gravity, parity, augmentation of labor, perineum results, episiotomy, position of the woman at birth, neonate birth weight, type of birth, and type of instrument used (vacuum, forceps, or none) were taken from the MBC database and were included in the analysis.. Participant hospitals had the same epidural analgesia regime for labor analgesia: 0.0625\% bupivacaine plus $2 \mathrm{mg}$ of fentanyl with Patient Controlled Epidural Analgesia (PCEA) for. Finally, for this study women who did not use EA as pain relief method during labor and caesarean sections were excluded, as our main goal was to analyse SPL after vaginal birth in women using EA.

The main outcome variable in this study was severe perineal laceration (SPL), involving the anal sphincter (third and fourth degree), according to the standardized Royal College of Obstetricians and Gynaecologists obstetric laceration classification (RCOG, 2015). Due to the low incidence of SPL, and that the evidence shows that episiotomy can lead to SPL (Escuriet R., 2017) it was decided to separate the results of the variables "perineum results" in three different categories:

Non $3^{\text {rd }}-4^{\text {th }}$ degree perineal laceration (it included intact perineum and $1^{\text {st }}-2^{\text {nd }}$ degree perineal tear).

Episiotomy (and episiotomy plus extended $1^{\text {st }} 2^{\text {nd }}$ degree perineal laceration).

$3^{\text {rd }}-4^{\text {th }}$ degree perineal laceration, (and episiotomy plus extended to $3^{\text {rd }}-4^{\text {th }}$ degree perineal laceration). 
The main exposure variable was use of $E A$ during vaginal birth (EA vs. non-EA). Instrumental birth was a secondary exposure variable. We segregated the data by instrumental birth and spontaneous vaginal birth, as vaginal birth is highly associated with episiotomy and perineal laceration (Anim-Somuah, 2018). This segregation helped to identify risk factors as well as protective factors in each cohort of women (instrumental and spontaneous vaginal births).

\section{Instrument development and data-collection}

Data was recorded by midwives in each participant hospital using a pre-designed standardized data collection sheet and data was introduced into the MCB electronic database. Information was obtained from the $\mathbf{3 0}$ maternity units in the four regions within Catalonia, Spain. Each midwife registered data from all childbearing women with uncomplicated pregnancies who met the study criteria, until a representative sample was achieved for each hospital. Detailed data was forwarded on a daily basis from the medical records system to the electronic MCB database, which contains information from July 2016 onwards.

\section{Statistical analysis}

We compared maternal, birth and foetal factors by "use" or "no use" of EA during labor. In addition, analysis of risk factors for SPL were stratified by mode of vaginal birth (noninstrumental versus instrumental birth) since the use of instruments, as showed in the literature, is linked to the performance of an episiotomy (Hudelist, 2008).

Descriptive analysis of the sample used percentages and frequency distributions for categorical variables and means (SD) for quantitative continuous variables. Univariate analysis was used to check the association of obstetric characteristics with the presence/absence of severe laceration. Categorical variables were compared by Chi-square or Fisher exact test, as appropriate, and quantitative variables by Student's t-test. Considering the dichotomous outcome (presence/absence of severe laceration), logistic regressions were performed in order to determine which of the covariates were associated with SPL. These associations were expressed by odds ratios (OR). Univariate and multivariate analysis were used to examine associations between the outcome and 
covariates. $P$ values less than 0.05 were considered as statistically significant. Statistical analysis was performed using STATA version 15 (STATA Corp., Texas, USA).

\section{Ethical considerations}

This study was approved by the ethics committee of the coordinating centre (Clinical Research Ethics Committee of Parc de Salut Mar 2017/7590/I) and later by the ethics committee of each participating centre. Ethics-committee approval was required for each participating hospital. Informed consent from women was not required because no intervention other than usual care was performed, and only anonymised data was collected. However, some settings decided to seek informed consent from women.

\section{FINDINGS}

The characteristics of the study sample are described in Table 1. Women who received epidural analgesia (EA) were more likely to be primiparous $(50.4 \%$ vs. $20 \%$ in the multiparous women group, p0.001), and this group of women also had more obstetric interventions during labor and birth: they were more likely to have an induction of labor (24.4\% vs. $10.98 \%$, p0.001), use oxytocin for augmentation (74.9\% vs. $34.8 \%$, p0.001), use of the lithotomy position at birth $(24.4 \%$ vs. $10.98 \%$, p0.001), more likely to have episiotomy (39.1\% vs. $15.2 . \%$, p0.001) and an instrumental birth (9.7\% vs. $1.85 \%$, p0.001), all having statistical significance.

4,258 (77.5\%) women used EA during labor and 1,239 (22.5\%) did not use it. Users of EA were younger than no users (30.7 years old vs. 31.2 years old in the no EA users' group, p. 0.003), having statistical significance. We observed a steady increase in use of EA as gestational age progressed (p0.001).

$3,128(73.5 \%)$ of women who had oxytocin for augmentation used EA, and $253(20.4 \%)$ women having oxytocin did not used EA.

$89.6(1.6 \%)$ of all women suffered severe perineal laceration (SPL). From the "no use of EA" group, 16 (1.3\%) women suffered SPL, and 72 (1.7\%) women who used EA suffered SPL, (p: 0.324), not being statistically significant.

$3,393(79,8 \%)$ women had spontaneous vaginal birth and used EA, 865 (28.4\%) women had instrumental birth and use EA, and 23 (1.85\%) women had instrumental birth and did 
not use EA, (p0.001); which appears highly significant. Vacuum extraction was used in $8.1 \%$ of the instrumental births, while the rate of forceps was $8.13 \%$, and low cavity forceps extraction a rate of $4.09 \%$.

Regarding maternal position at birth, 3,190 (74.92\%) women who used EA and 432 (34.87\%) women who did not use EA adopted the lithotomy position. From the 1,875 (34.1\%) women who adopted an alternative position for birth, 807 (65.1\%) did not use EA, (p: 0.000). 4,258 (77.5\%) women used EA and 1,239 (22.5\%) of women did not use EA, which is highly significant, ( $\mathrm{p}: 0.000)$.

We analysed data by spontaneous birth versus instrumental birth (Table 2). 32 (0.9\%) women with spontaneous vaginal birth and EA use had SPL and 14 (1.1\%) women with spontaneous vaginal birth who did not use EA had SPL, (p: 0.531). Moreover, 40 (4.6\%) women who had instrumental birth with EA and 2 (8,7\%) women who had instrumental birth with without EA had SPL (p: 0.364), not being statistically significant.

We saw that $34(2 \%)$ primiparous women in the spontaneous birth group suffered SPL, and $12(0.4 \%)$ multiparous women in this group did so too, ( $p: 0.000)$, showing multiparity is a strong protector for SPL. On the other hand, 34 (5\%) primiparous women in the instrumental birth group suffered SPL versus 3.9\% of multiparous women ( $p: 0.514)$, this was no longer statistically significant.

$18(1.6 \%)$ women with spontaneous vaginal birth with episiotomy and $0.8 \%$ with spontaneous vaginal birth without episiotomy had SPL, ( $p: 0.025)$, being this result strongly significant and showing episiotomy as a strong risk factor for SPL. On the other hand, 29 $(4,1 \%)$ women who had instrumental birth with episiotomy and $13(7.2 \%)$ women who had instrumental birth without episiotomy suffered SPL, (p: 0.078). Therefore, episiotomy performed in the context of an instrumental birth ceases to be a significant risk factor for SPL.

27 (1\%) women who had spontaneous vaginal birth with oxytocin suffered SPL versus 19 $(1 \%)$ women who had spontaneous vaginal birth without oxytocin, ( $p: 0.808)$. In the instrumental birth group with oxytocin augmentation 37 (4.9\%) of women sustained SPL versus $5(3.8 \%)$ of those who did not use oxytocin ( $p: 0.594)$.Oxytocin is thus not a statistically significant risk factor regardless of the mode of birth.

$16(0.9 \%)$ women with spontaneous vaginal birth who did not use the lithotomy position at birth had SPL compared to $30(1.1 \%)$ who had a spontaneous birth and adopted the 
lithotomy position. Those who adopted an alternative position had less risk of developing SPL (p: 0.535), although not statistically significant.

821 (92.5\%) women had instrumental birth in lithotomy and 36 (4.4\%) of these women had SPL. And 66 (7.4\%) women had instrumental birth in a different position and 6 (8.97\%) had $\mathrm{SPL}$, not being statistically significant.

Regarding birth weight and risk of SPL we notice a positive correlation between birth weight and SPL in the instrumental birth group, whereby lower birth weights result in lower rates of SPL, $23(5.7 \%)$ women with babies with a birth weight between 3,001 and 3,500 grams had SPL, and how 6 (13.6\%) women with babies with a birth weight above 4,000 grams had SPL. This is double the rate and is statistically significant.

We conducted a logistic regression (Table 3) to see the impact of each variabe in each type of birth (spontaneous/ instrumental birth). We found that the use of EA was not a risk factor for SPL neither in spontaneous nor in instrumental birth (OR $0.47 \mathrm{Cl} 95 \%$ : 0.21-1.02, p: 0.060 ; OR $0.45 \mathrm{Cl} 95 \% 0.94-2.11, \mathrm{p}: 0.310$, respectively), although this is again not statistically significant.

Use of oxytocin in spontaneous and in instrumental vaginal birth group was found to be a risk factor for SPL (OR: 0.94; IC 95\%: 0.46-1.90, p: 0.853 and OR 1.34; IC: 95\% 0.49-3.64, p: 0.565$)$ respectively, and it is not statistically significant.

Episiotomy was a significant risk factor for SPL in both groups (spontaneous vaginal birth and instrumental birth) (OR 1.2; Cl 95\% 0.65-2.32, p: 0.522 vs. OR 0.53; Cl 95\%: 0.26-1.08, p:0.083), respectively.

Multiparity was a strong protector in spontaneous birth for SPL and this is statistically significant (OR 0.17; 95\% Cl 0.81-0.34, p:0.000). However, multiparty in instrumental birth loses this significance (OR 0.63; 95\% $\mathrm{Cl} 0.27-1.43$, p: 0.274$)$.

The lithotomy position on the other hand was not a risk factor for SPL in neither of the groups (OR 1.28; Cl 95\% 0.65-2.52, p: 0.467 vs. OR 0.55; Cl 95\%: 0.21-1.40, p: 0.212), respectively.

\section{DISCUSSION}


In this study, epidural analgesia (EA) was not associated with increased risk of SPL. When confounders such as augmentation, episiotomy, parity, lithotomy position, birth weight and gestational age were included in the analysis, EAwas no longer associated with SPL, suggesting that other factors determine their interrelation. Identifying individual factors that may lead to SPL is difficult because in most women many risk factors seem to interact. In our study, instrumental birth and primiparity were the strongest associated risk factors for SPL. The incidence rate of SPL during childbirth was $1.63 \%$, similar to the incidence described in different countries (0.8\% - 3\%) (Loewenberg-Weisband et al., 2014 and Edqvist et al., 2017), although the rate of EA use was 77\%, much higher than in other countries; [(e.g. 30\% in the United Kingdom, 60\% in the USA, 30-69\% in Canada (Anim-Somuah et al, 2011; Grant et al, 2015)]. It is plausible to assume that this high rate of EA use is at least partly attributable to the medical obstetric practice that is predominant in Catalonia and which tends to be highly interventionist (Schytt et al., 2010).

As previous studies show, our data identifies instrumental birth as significantly more prevalent among women with SPL (Pergialiotis et al., 2014). Therefore in the multivariate analysis we decided to segregate between spontaneous and instrumental birth, to identify which factors influence independently in each type of birth on the incidence of SPL. With this segregation, the results showed that EA use was not a primary factor for SPL neither in instrumental nor in spontaneous birth, as demonstrated in different studies (LoewenbergWeisband et al., 2014; Landy et al., 2011). On the other hand (and this is the most significant finding of our study), the logistic regression found that primiparity was clearly associated with the highest rates of SPL. Primiparity as a risk of SPL has been amply described in the literature (Groutz et al., 2011; Pergialiotis et al., 2014). One of the explanations for this association, is the lack of elasticity of the perineum in primigravida women (Simic et al., 2012). On the other hand, multiparity was a strong protector against SPL in spontaneous vaginal birth, but multiparity did not protect in instrumental births. This association could be explained by the damaging effect to the perineum by the instrument itself independently to parity (Simic et al., 2012, Hsieh et al., 2014).

Parity is not a modifiable factor, however, other studies have shown that certain factors that contribute to the occurrence of SPL are modifiable (Hsieh et al., 2014; Smith et al., 2013). Among them, the factors that are easiest to modify include the use of the "Hands-off" approach for perineal control during labor. This has shown a positive effect on the mother's health because of the reduction of episiotomy and third degree tearing (Rezaei et al., 2014). The use of warm pads (Aasheim et al., 2017) during the second stage of labor have also 
been proven in reducing the incidence of SPL as demonstrated in the Cochrane Database Systematic Review (Aasheim et al., 2017; Geranmayeh., 2012). Being respectful of the pace of labor may contribute towards protecting against SPL in multiparous women. Respecting the progress of labor without intervening unnecessarily, even in those circumstances when progress may be slower than expected can help to avoid instrumental births, in which scenario multiparity loses its protection for SPL.

Many studies have shown EA use during labor causes prolonged second stage (Rognant et al., 2012; .Borello-France et al., 2006,), which usually culminates in an instrumental birth with an episiotomy on many occasions [a risk factor of SPL on its own (Carolli et al., 2014; Escuriet et al., 2017]). Therefore, EA contributes to the causal chain of events leading to SPL (Lothian J., 2014).

Our results showed that women with spontaneous births without episiotomy sustained lower rates of SPL than when an episiotomy was performed. However, an episiotomy protects against SPL during instrumental vaginal births, which correspond to the findings of different studies (De Vogel et al., 2012, De Leeuw et al., 2008, Simic et al. 2012 ). This can be explained by the fact that the episiotomy is performed in an instrumental birth to widen the diameter of the introitum and thus reduce the potential trauma the instrument can cause (specially forceps).

Augmentation of labour, was associated with higher use of EA. Augmentation and episiotomy are modifiable risk factors also, and the literature recommends that efforts should be made to use these procedures only when medically necessarily (Landy et al., 2011) as many common obstetric practices including the above, are of limited or uncertain benefit for low-risk omen in spontaneous labor (ACOG, 2017).

In our study, $74.9 \%$ of primiparous women were administered oxytocin. Recent studies report an increasing use of oxytocin, especially among primiparous women, with a prevalence of use reported to be $37-75 \%$. This high rate of augmentation with oxytocin in our study may be partly explicated by an over-use of the procedure, employed even in the absence of medical indications. Therefore, guidelines for labor dystocia and augmentation, based on the latest evidence should be published, allowing labor to progress uninterrupted, and respecting its normal progress without rushing it, especially in view of Gaudernack et al.'s study which shows the association between use of oxytocin and instrumental births (Gaudernack et al., 2018). Therefore oxytocin should be used with caution and only when medically indicated. This is perhaps particularly important in multiparous women, as 
interfering with the normal process of labor in the absence of medical indication can lead to instrumental births in which case, multiparity ceases to be a protective factor for SPL.

Our study showed higher rates of SPL in women who adopted the lithotomy position, that is also a modifiable factor. Moreover, the "lithotomy position" group used more EA, similar to other studies (Dahlen et al., 2007). We found that alternative positions to lithotomy was associated with lower rates of instrumental births and SPL (Elvander et al., 2015). Childbearing women using EA in our study showed to be more likely to labor on the bed, to give birth in lithotomy and be more immobile, when compared to women without EA. World Health Organization encourages freedom to move and to choose a comfortable position for labour and birth (WHO, 2018).

Within this backdrop in Catalonia, it is welcome that the Strategy for Assistance at Normal Childbirth recommends a more "woman-centred" model of care which claims respect for the natural process of labor rejecting any intervention unless this is deemed necessary. However, it seems that these recommendations are not always implemented, and a highly interventionist approach to care is still practised in many of the Catalan obstetric units. This may be partly due to midwives not having the appropriate training to support a more "womancentred" model of care. As a consequence of the assessment, and despite the high EA use and high intervention rate in general, Catalonia keeps working for a more "woman-centred" model of care and aims to improve maternity results. One of the examples is that the first birth centre in Catalonia was opened in November 2017, Martorell Birth Centre, and more birth centres are planned. Also, new guidelines on clinical practice for care in normal

childbirth are to be published.

This study has identified risk factors relating to obstetric and midwifery practices, which have the potential to minimize SPL rate and EA use. The clinical information derived from our study is a major strength as it provides an insight into current obstetric practice in a large and diverse population.

\section{LIMITATIONS}

The overall rate of SPL was $1,6 \%$. This low incidence prevents us from drawing any firm conclusions. 
Future studies, should consider different variables such as EA dose, type of epidural drugs used, length of second stage of labour, timing of EA exposure and others. These will help discern how use of EA during labour interferes with the process of labor, and it will identify strategies that could help continue reducing the rate of SPL and improving birth outcomes. In addition, management of labor at the 30 hospitals in the study region may differ from that of other regions, thereby limiting the generalizability of our findings.

\section{$\begin{array}{llll}\text { IMPLICATION } & \text { FOR } & \text { PRACTICE } & \text { AND } \\ \end{array}$}

This study provides incidence rates of SPL in our setting and identifies various risk factors and practice areas which contribute to SPL during childbirth. These data corroborate that use of instruments and primiparity increase the frequency of SPL, therefore, efforts should be made to minimise instrumental births and to think about primiparity as a risk by its own so special attention should be paid to them.

The main finding seen in this study is the overuse of EA in Catalonia. Even though this is not a direct cause of SPL, it is part of the cascade of interventions that may lead to it and so it should be avoided whenever possible. This agrees with the recommendations by the Lancet's Maternal Health series, which highlights the detrimental effects of overusing technology in developed countries (McDougall et al, 2016). Since EA has been long known to be a predictive risk factor for women's health problems such as increased risk of SPL, its overuse appears to be in contradiction to the new Wold Health Organization recommendations (WHO, 2018).

Our findings show the need for new educational programs for professionals in maternity care in Catalonia, to promote and support physiological birth. This educational program should include an emphasis on providing midwives with the necessary skills and competences to support birth physiology without resorting to routine obstetric practices that are of limited benefit for the vast majority of women.

Moreover, maternity health programs should include the promotion and real accessibility to alternative pain relief methods, (pharmacological and non-pharmacological) aside EA. This study should also serve practitioners, in offering sound evidence on which to base hospital's guidelines in regard to facilitating physiological birth, encouraging birth in alternative positions to lithotomy. It should encourage professionals to re-evaluate the role they play in women choosing EA during labor, as professionals do influence the woman's decisionmaking (Schytt and

Waldenstrom,

2010). 
It ultimately should encourage obstetricians in regard to the use of instruments to aid the birth, to weight the risk and benefits before performing this type of birth, especially if the goal is to reduce the risk of maternal SPL and to improve maternal health outcomes.

Last but not least, this study identifies the need for policy changes and for new guidelines to protect pregnant women and to promote normal and physiological labor. Since support from care givers reduces the need for analgesia in labor, one to one midwifery care should be promoted (Hodnett et al., 2012). Our findings should prompt the implementation of preventive strategies to reduce the likelihood of SPL. Most pregnant women are followed up in the antenatal clinic and in maternity units by their midwives and adding information about how to prevent SPL during childbirth and information about pain relief methods during labor, should be feasible and affordable.

In conclusion, there appears to be a need to change the philosophy of maternity care provision among Obstetricians and Midwives from one that is medically focused to one that is woman-centred. Ultimately, this philosophy and belief on the birthing process without any unnecessary intervention needs to be relayed to women and to Catalan society.

\section{CONFLICT OF INTEREST}

None.

\section{REFERENCES}


Anim-Somuah, M, Smyth, R, Cyna, M, Cuthbert, A. Epidural versus non-epidural or no analgesia for pain management in labour. Cochrane Database Systematic Reviews. 2018, May 21;5.

Dudding, T, Vaizey, C, Kamm, M. Obstetric Anal Sphincter Injury: Incidence, Risk Factors, and Management. Annals of Surgery: February 2008 - Volume 247 - Issue 2 - p 224-237.

Edqvist, M, Hildingsson, I, Mollberg, M, Lundgren, I, Lindgren, H. Midwives' Management during the Second Stage of Labor in Relation to Second-Degree Tears-An Experimental Study. Birth, 2017; 44:1.

Elvander, C, Ahlberg, M, Thies-Lagergren, L, Cnattingius, S, Stephansson, O. Birth position and obstetric anal sphincter injury: a population-based study of 113,000 spontaneous births. BMC Pregnancy Childbirth, 2015; 15: 252.

Escuriet, R, García-Lausin, L, Salgado-Poveda, I, Casañas, R, Robleda, G, Canet, O, Perez-Botella, M, Frith, L, Daly, D, Pueyo, M, 2017. Midwives'contribution to normal childbirth care: Cross-sectional study in public health settings, the MidconBirth Study Protocol. European Journal of Midwifery, 1:4.

Escuriet, R, Pueyo, MJ, Garcia-Lausin L, Obregón N, Pérez-Botella M, López JM, SalgadoProveda, I, Marínez-Pascual, M, Asin-Chinchilla, P , Santos-Viva, M, León-

Escuriet, R, Pueyo, M J, Perez-Botella, M, Espada, X, Salgado, I, Gómez, A, Biescas, H, Espiga, I, White, J, Fernandez, R, Fust, J, Ortn, V , 2015. Cross-sectional study comparing public and private hospitals in Catalonia: is the practice of routine episiotomy changing?. BMC Health Services Research, 15 (95). 
Grant, E, Tao, W, Craig, M, Mc Intire,D, Leveno, K. Neuraxial analgesia effects on labour progression: facts, fallacies, uncertainties and the future. British Journal of Obstetrics \& Gynaecology, 2014 Published Online 4 August .

Greve, T, Lundbye-Christensen, S, Nickelsen, CN, Secher, NJ. Maternal and perinatal complications by day of gestation after spontaneous labor at $40-42$ weeks of gestation. Acta Obstetricia et Gynecologica Scandinavica, 2011; 90(8):852-6.

Groutz, A, Hasson, J, Wengier, A, Gold, R, Skornick-Rapaport, A, Lessing, J. Third and fourth-degree perineal tears: prevalence and risk factors in the third millennium. American Journal of Obstetrics and Gynaecology ,2011; 204 (4): 347.e1-4.

Gurol-Urganci, D, Edozien, T, Adams, D, Richmond, A, Templeton, J. Third and fourthdegree perineal tears among primiparous women in England between 2000 and 2012: time trends and risk factors. British Journal of Obstetrics \& Gynaecology, 2013,Volum 120, issue 12.

Hasegawa, J, Farina, A, Turchi, G, Hasegawa, Y, Zanello, M, Baroncini, S. Effects of epidural analgesia on labor length, instrumental delivery, and neonatal short-term outcome. Journal of Anaesthesia, February 2013, Volume 27, Issue 1, pp 43-47.

Hawkins, J. Epidural analgesia for labor and delivery. New England Journal of Medicine. 2010 Apr 22;362(16):1503-10.

Hodnett, E, Gates, S, Hofmeyr, J, Sakala, C. Continuous support for women during childbirth. Cochrane Database Systematic Review 2012; 10: CD003766.

Hudelist G, Mastoroudes H, Gorti M, 2008. The role of episiotomy in instrumental delivery: is it preventative for severe perineal injury? Journal of Obstetrics and Gynaecoly, 28(5): 46973. 
Jones, L, Othman, M, Dowswell, T, Alfirevic, Z, Gates, S, Newburn, M, Jordan, S, Lavender, T, Neilson, JP, 2012. Pain management for women in labour: an overview of systematic reviews. Cochrane Database Systematic Reviews. 14;(3).

Kalis, V, Stepán, J Jr, Králíckova, M, Zluvová, P, Rokyta, Z. Maternal position at the delivery and perineal trauma. Ceska Gynekologie Journal, 2007, 72(4):241-6.

Kibuka, M, Thornton, J. Position in the second stage of labour for women with epidural anaesthesia. Cochrane Systematic Review - Intervention Version published: 24 February 2017.

Landy, H, Laughon, S, Bailit, J, Kominiarek, A, Gonzalez-Quintero, H, Ramirez, M, Haberman, S, Hibbard, J, Wilkins, I, Banch, W, Gregory, K, Hoffman, MK, Learman, A, Hatjis, C, Vanveldhuisen, C, Reddy, M, Troendle, J, Sun, L, Zhang, J. Characteristics associated with severe perineal and cervical lacerations during vaginal delivery. Journal of Obstetrics \& Gynaecology, 2011, 117:627-35.

Larios, F, White, J, and Participants in the MIDCONBIRTH Study, 2017. Vaginal Delivery Care, Episiotomy Performance and Examination of Severe Perineal Tears: Cross-Sectional Study in 43 Public Hospitals. Obstetrics \& Gynecology International Journal. Volume 7 Issue $6-2017$.

Loewenberg-Weisband, Y, Grisaru-Granovsky, S, loscovich, A, Samueloff, A, CalderonMargalit, R, 2014. Epidural analgesia and severe perineal tears: a literature review and large cohort study, The Journal of Maternal-Fetal \& Neonatal Medicine, 27:18, 1864-1869, DOI: 10.3109/14767058.2014.889113.

Lothian, J. Healthy Birth Practice \#4: Avoid Interventions Unless They Are Medically Necessary. Journal of Perinatal Education, 2014 ; 23(4): 198-206. 
Mc. Dougall, L, Campbell, OMR, Graham, W. Maternal Health: an executive summary for the Lancet's series. The Lancet; September 2016.

Meister, M, Cahill, A, Cannor, S, Woolfolk C, Lowder, J. Predicting obstetric anal sphincter injuries in a modern obstetric population. American Journal of Obstetrics and Gynaecology, 2016 Sep;215(3):310.e1-7.

Ministry of Health and consumer's affairs, 2007. Strategy for Assistance at Normal Childbirth in the National Health System. Madrid, Spain.

Ministry of Health and consumer's affairs, 2012. Report on care for childbirth in the National Health System in Catalonia. Catalonia, Spain (not published).

Nkwabong, E. Maternal and neonatal complications of macrosomia. Epub, 2014; 44(4):2014.

Pergialiotis, V , Vlachos, D, Protopapas, A, Pappa, K, Vlachos, G. Risk factors for severe perineal lacerations during childbirth. International Journal of Gynaecology and Obstetrics, International Journal of Gynaecology and Obstetrics, 2014, 125; 6-14.

Prager, M, Andersson, K, Stephansson, O, Marchionni, M, Marions, L. The incidence of obstetric anal sphincter rupture in primiparous women: a comparison between two European delivery settings. Acta Obstetricia et Gynecologica Scandinavica, 2008, 87:209-215.

Rathfisch, G, Dikencik, B, Kizilkaya, N, Comert, N, Tekirdag, A, Kadioglu, A. Effects of perineal trauma on postpartum sexual function. Journal of Advanced Nursing, 2010, 66(12), 2640-2649. 
Rognant, S, Benoist, G, Creveuil, C, Dreyfus, M. Obstetrical situations with a high risk of anal sphincter laceration in vacuum-assisted deliveries. Acta Obstetricia et Gynecologica Scandinavica, 2012, 91:862-868.

Royal College of Obstetricians and Gynaecologist. Third- and Fourth-degree Perineal Tears, Management.Green-top Guideline No. 29. 2015.

Simic, M, Cnattingius, S, Petterson, G, Sandström, A, Stephansson, O. Duration of second stage of labour and instrumental delivery as risk factors for severe perineal lacerations: population-based study. BMC Pregnancy and Childbirth, 2017, 17:72.

Schytt, E and Waldenstrom, U. Epidural analgesia for labour pain: whose choice? Acta Obstetricia et Gynecologica, 2010; 89: 238-242.

Smith, L, Price, N, Simonite, V, Burns, E. Incidence of and risk factors for perineal trauma: a prospective observational study. BMC Pregnancy Childbirth, 2013 Mar 7;13:59.

Sng, B, Leong, WL, Zeng, Y, Siddiqui, FJ, Assam, PN, Lim, Y, Chan, ES, Sia, AT. Early versus late initiation of epidural analgesia for labour. Cochrane Database Systematic Review. 2014 Oct 9;(10).

Voldner, N, Froslie, K, Haakstad, L, Bo, K, Henriksen, T. Birth complications, overweight, and physical inactivity. Acta Obstetricia et Gynecologica Scandinavica. 2009,88:550-555.

World Health Organization, 2018. Making childbirth a positive experience guideline on intrapartum care. 